Artículo original

\title{
Viabilidad y germinación in vitro de taxones de las tribus Cymbidieae y Epidendreae (subfamilia Epidendroideae, Orchidaceae)
}

\author{
Adriana Paola Bonilla-Sánchez*, Hilda Rocío Mosquera-Mosquera \\ Grupo de Genética y Biotecnología vegetal y Microbiana, Universidad del Tolima (GEBIUT), Ibagué, Colombia
}

\begin{abstract}
Resumen
Las orquídeas Epidendroideae presentan el polen organizado en polinios con diferente grado de compactación; cada polinio puede contener hasta 4 millones de granos de polen. En este estudio se evaluó la viabilidad y la germinación in vitro del polen aglutinado en polinios. Se evaluó la calidad polínica de siete taxones en dos tribus utilizando métodos palinológicos ex vitro (tinción con acetocarmín glicerol al $2 \%$ ) y directos (germinación in vitro) en tres medios, M1, M2 y M3. La viabilidad con acetocarmín fue superior al $85 \%$ en los siete taxones estudiados. El mayor porcentaje de germinación in vitro se obtuvo en Catasetum tabulare, con un $16 \%$; en el resto de taxones los valores oscilaron entre el $2 \%$ y el $5 \%$ a las 72 horas de observación. El medio M3 fue el más exitoso, con 18 y $20 \%$ de germinación en los taxones estudiados. Aunque la viabilidad del polen es alta, presenta tasas muy bajas de germinación, probablemente por el alto grado de compactación y la ausencia de aperturas, lo que la hace lenta y poco efectiva en medios artificiales. Los resultados ratificaron que no todo el polen viable germina, y que existe un alto grado de especificidad y dependencia frente a las sustancias estimuladoras de la germinación del polen provenientes del estigma de las orquídeas, lo que hace necesario seguir profundizando en el análisis de la biología del polen de la subfamilia Epidendroideae para comprender un poco más sus procesos de multiplicación natural.
\end{abstract}

Palabras clave: Biología polínica; Polinio; Tétradas; Tubo polínico.

Viability and in vitro germination of taxa from Cymbidieae and Epidendreae tribes (subfamily Epidendroideae, Orchidaceae)

\begin{abstract}
The pollen in Epidendroideae orchids is organized in pollinia with different degrees of compaction; each pollinium can contain up to 4 million pollen grains. In the present study, we analyzed the effect of the compaction of the pollinium on the viability and germinative capacity of the pollen. We evaluated the pollen quality of seven taxa from two tribes using ex vitro palynological methods (staining with acetocarmine glycerol $2 \%$ ) and direct methods (in vitro germination) in three media: M1, M2, and M3. Viability with acetocarmine was over $85 \%$ in the seven taxa studied. The highest percentage of in vitro germination was obtained in Catasetum tabulare with $16 \%$; in the rest of the taxa, the values oscillated between $2 \%$ and $5 \%$ at $72 \mathrm{~h}$ of observation. The M3 medium was the most successful with $18 \%$ and $20 \%$ germination in the taxa studied. Although the viability of pollen is high, it has very low germination rates probably affected by the high degree of compaction and the absence of openings, which makes germination in artificial media slow and ineffective. The results confirm that not all viable pollen can germinate and that there is a high degree of specificity and dependence on the stimulating substances for the germination of pollen present in the stigma of the orchids, which makes it necessary to continue deepening in the analysis of the pollen biology of the subfamily Epidendroideae to understand a little more its processes of natural multiplication.
\end{abstract}

Key words: Pollen biology; Pollen tube; Pollinium; Tetrads.

\section{Introducción}

Los miembros de la subfamilia Epidendroideae, incluidos Cymbidieae y Epidendreae, se caracterizan por exhibir flores hermafroditas y zigomorfas (Díez, 2001), en las cuales el atributo más notable es una estructura conocida como columna, conformada por la fusión del androceo y el pistilo, que resulta en la presencia de un solo estambre con granos de polen agregados de diversa forma que reciben el nombre de polinios (Dressler, 1981). El polinio se puede definir como un conglomerado de tétradas polínicas con diferentes

\section{*Correspondencia:}

Adriana Paola Bonilla Sánchez; apbonillas@ut.edu.co

Recibido: 6 de mayo de 2019

Aceptado: 8 de julio de 2019

Editor: Elizabeth Castañeda 
grados de cohesión, que puede albergar alrededor de 4 millones de granos de polen por unidad (Damon \& Nieto, 2012; Johnson \& Edwards, 2000). Pueden tener textura granular, séctil o compacta (Hesse, et al., 1989; Dressler, 1993; Freudenstein \& Rasmussen, 1997; Johnson \& Edwards, 2000; Rothacker, 2007), o pueden ser enteros o bipartidos (con sutura) (Mosquera, et al., 2019). El polinio es dispersado desde la antera por el polinizador y puede depositarse como una unidad completa en el estigma (debido a su compactación) o separarse por másulas cuando su textura es séctil (Singer, et al., 2008).

Si bien el análisis de la estructura del polinio permite interpretar el proceso de dispersión del polen, no es fácil determinar sí su compactación dentro del polinio incide en el porcentaje de su viabilidad y germinación, además de las posibles repercusiones en el proceso de fecundación del óvulo. Por ello, las herramientas biotecnológicas agrupadas en métodos directos in vitro y las técnicas indirectas basadas en parámetros fisiológicos, como el color de la reacción bioquímica por el uso de gelatina de acetocarmín glicerol, son primordiales para determinar la calidad del grano de polen (Aramediz, et al., 2013), de lo cual dependen fenómenos como la polinización, el mejoramiento genético, los procesos de reconocimiento polen-pistilo, la fertilización, etc. (Rejón, 2010).

El objetivo de este estudio consistió en evaluar la viabilidad y la germinación in vitro del polen aglutinado en polinios en siete taxones pertenecientes a las tribus Cymbidieae y Epidendreae. Los resultados obtenidos han permitido reconocer las estrategias de dispersión y reproducción de las cuales hacen uso estas especies. Los métodos estandarizados en el laboratorio son una herramienta útil para la conservación del polen de parentales empleados en procesos de propagación ex vitro. Por tal razón, la información que se presenta aquí constituye en un aporte valioso para la comprensión de la enorme diversidad, variabilidad y amplia distribución de la subfamilia Epidendroideae.

\section{Materiales y métodos}

Área de estudio y sitios de muestreo. El estudio se desarrolló en tres puntos fitogeográficos de la ciudad de Ibagué. La primera zona correspondió a la Fundación Orquídeas del Tolima (Reserva de la Sociedad Civil), donde se recolectó la mayor parte de las muestras florales, situada a $4^{\circ} 25^{\prime} \mathrm{N}$ y $75^{\circ} 15^{\prime} \mathrm{O}$ en una zona de vida de bosque premontano bajo, con una temperatura promedio de $24{ }^{\circ} \mathrm{C}$, una altitud de $1.321 \mathrm{~m}$ s.n.m. y una precipitación promedio anual de 1.993 $\mathrm{mm}$. El segundo punto de muestreo fue el Jardín Botánico San Jorge, reserva natural situada a $4^{\circ} 26^{\prime} \mathrm{N}$ y $75^{\circ} 13^{\prime} \mathrm{O}$ en una zona de formación de bosque premontano bajo, con una temperatura promedio de $24{ }^{\circ} \mathrm{C}$, una altitud de 1.133 m s.n.m. y una precipitación promedio de $1.993 \mathrm{~mm}$. El último punto de recolección comprendió al área boscosa de El Silencio (bosque premontano alto), perteneciente al área rural del Cañón del Combeima (Ibagué), situado a $4^{\circ} 36^{\text {' }}$
$\mathrm{N}$ y $75^{\circ} 19^{\prime} \mathrm{O}$, con una temperatura promedio de $18{ }^{\circ} \mathrm{C}$, una altitud de $2.586 \mathrm{~m}$ s.n.m. y una precipitación promedio oscilante de $1.816 \mathrm{~mm}$.

Material estudiado. Se analizaron los polinios frescos recolectados a partir de flores en estado de antesis de siete taxones (Catasetum tabulare Lindl., Oncidium sp., Anguloa clowesii (Ruiz \& Pav.) Lindl., Gongora sp.) en la tribu Cymbidieae y (Cattleya trianae Linden \& Rchb.f., Epidendrum ciliare L. y Epidendrum sp.) en la tribu Epidendreae, de la subfamilia Epidendroideae.

Métodos palinológicos. Para determinar la calidad del gametofito masculino, se evaluaron dos técnicas de análisis, una ex vitro (viabilidad) y otra in vitro (germinación), de la siguiente manera.

Determinación de la viabilidad del polinio. En el análisis de la viabilidad se empleó la metodología propuesta por Ordóñez (2014), la cual tiene como fundamento la coloración del contenido protoplasmático de los granos de polen. Los polinios contienen tétradas, y para verificar la viabilidad, se tuvo en cuenta cada grano de polen que las conformaba, pues todos tenían el potencial de germinar. Los polinios fueron colocados en el portaobjetos con tres o cuatro gotas de acetocarmín glicerol al $2 \%$, y posteriormente se disgregaron hasta obtener una muestra homogénea. Esta preparación se dejó reposar durante un minuto, luego se cubrió con láminas cubreobjetos y, por último, se refrigeró a $4{ }^{\circ} \mathrm{C}$ en posición horizontal. Las muestras se leyeron bajo un microscopio de campo claro a $40 \mathrm{x}$ a partir de las 3 horas de su montaje. Se analizaron 500 granos de polen por muestra, equivalentes a 125 tétradas polínicas. El resultado se expresó como el porcentaje de granos viables comparado con el número total de granos de polen multiplicado por cien. Se consideró como viables aquellos granos que registraban una coloración rojo carmín intensa en el citoplasma, y como granos no viables, los que presentaban ausencia de coloración o irregularidades en su morfología.

Germinación del polen in vitro. Para evaluar la capacidad germinativa del polen, se emplearon tres medios de cultivo diferentes: el medio M1, de consistencia sólida, propuesto por Pritchard \& Prendergast (1989), y el medio M2, medio líquido que corresponde a la modificación del medio BK (Brewbaker \& Kwack, 1963) realizada por Tsai \& Chang (2010). El medio M3 fue diseñado por los autores para mejorar la respuesta germinativa in vitro: es sólido e incluyó el estigma de cada flor, agar al $1 \%$, agua destilada y pH igual a 5,7 y no contenía sacarosa. Una vez solidificado el agar, se agregó de manera uniforme la sustancia estigmática de cada especie en evaluación recogida en un pincel. Los medios de cultivo en estado sólido se vertieron en cajas de Petri (20 ml) y el medio en estado líquido se usó en portaobjetos. Los polinios fueron hidratados, macerados y luego depositados en cada medio de cultivo. Se analizaron 500 granos de polen por polinio, con 10 repeticiones por cada taxón.

Todos los tratamientos se incubaron en oscuridad a una temperatura que oscilaba entre 25 y $27^{\circ} \mathrm{C}$. La germinación 
se detuvo 72 horas después de la siembra agregando a cada medio 2 a 3 gotas de acetorcarmín glicerol al $2 \%$. Este colorante permitió la diferenciación de las tétradas y mejoró la observación de los tubos polínicos. Se consideraron como granos de polen germinados aquellos que emitieron un tubo polínico con una longitud mayor o igual al diámetro del polen. Por último, se contaron los granos de polen ger-minados en un mínimo de 10 campos ópticos, y los resultados se expresaron como el porcentaje de polen con posibilidad de germinar comparado con el número total de granos de polen por campo multiplicado por cien.

Análisis estadístico. Se utilizaron las pruebas de ShapiroWilk y Anderson Darling para determinar si los datos seguían una distribución normal. Para evaluar el nivel de significación con respecto a las diferencias en los porcentajes de viabilidad y germinación del polinio, se compararon las medianas empleando la prueba no paramétrica de KruskalWallis, y se consideraron significativos valores de $\mathrm{p}$ menores que $0,05(\mathrm{p} \leq 0,05)$. Tanto en las pruebas de viabilidad como de germinación, se contabilizó un total de 5.000 granos de polen $(500 \times 10$ repeticiones independientes), y en cada placa y caja de Petri se evaluaron 10 campos de observación óptica. Estos análisis se ejecutaron con el paquete estadístico R-Project, versión libre.

\section{Resultados}

Viabilidad del polinio. El método citológico para determinar la calidad del polen en orquídeas con polinios compactos usando acetocarmín glicerol al $2 \%$ evidenció altos niveles de viabilidad (Figura 1). Según los resultados, los mayores porcentajes de viabilidad se presentaron en los taxones Gongora sp. (93\%), Anguloa clowessi (91\%), C. tabulare (89\%) y Oncidium sp. (88\%). Dado que la prueba de Kruskal-Wallis no evidenció diferencias significativas al comparar las medianas de viabilidad, con un valor de p igual a 0,3971 y un alfa de 0,05 , es posible afirmar que los cuatro taxones evaluados en la tribu Cymbidieae tuvieron la misma disponibilidad de granos de polen viables, como se observa en la Figura 2A.

Por otra parte, en la tribu Epidendreae el mayor porcentaje de viabilidad se observó en la especie Cattleya trianae (94\%), seguida por Epidendrum ciliare y Epidendrum sp., con un 92 y $91 \%$ de viabilidad, respectivamente. Por su parte, la prueba estadística arrojó un valor de p de 0,2871 y un alfa de 0,05 , lo que permitió inferir que no hubo diferencias significativas al comparar las medianas de viabilidad polínica en estas tres especies (Figura 2B).

Germinación del polinio. En la tribu Cymbidieae el mayor porcentaje de germinación a las 72 horas de evaluación se presentó en la especie $C$. tabulare, con un $16 \%$ de germinación, a diferencia de los taxones $A$. clowessi, Gongora sp. y Oncidium sp., los cuales evidenciaron muy bajos porcentajes de germinación (4, 4 y 5\%, respectivamente), con un margen de error de 0,05 (Figura 3A). Los resultados con la prueba de Kruskal-Wallis arrojaron un valor de $\mathrm{p}$ igual

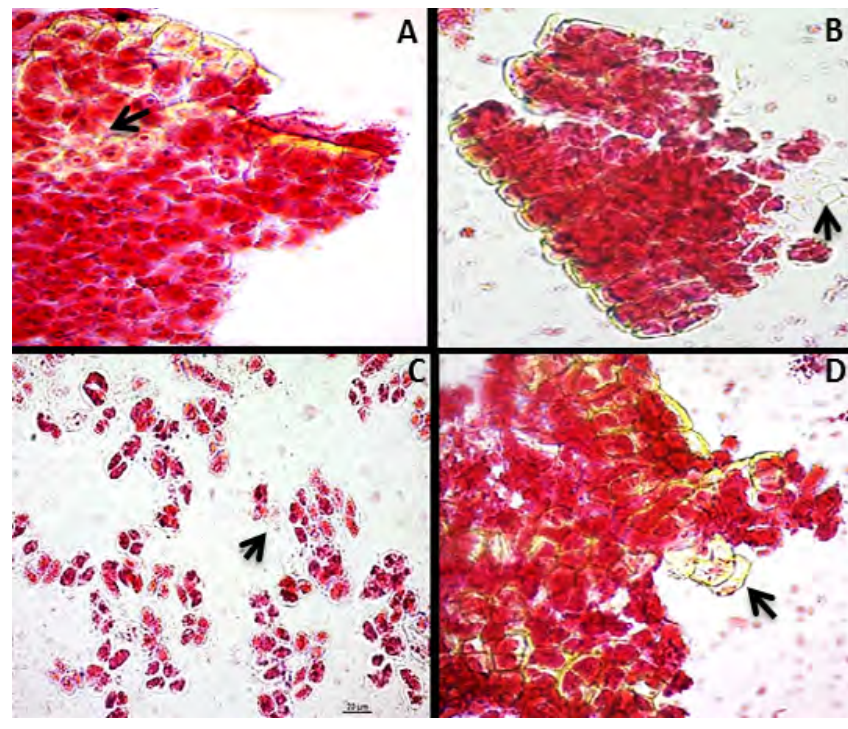

Figura 1. Tétradas polínicas en las que se observan granos de polen inviable (flecha) en contraste con el gran número de polen viable de los taxones con coloración rojo intenso: A) Catasetum tabulare. B) Oncidium sp. C) Cattleya trianae. D) Epidendrum sp.

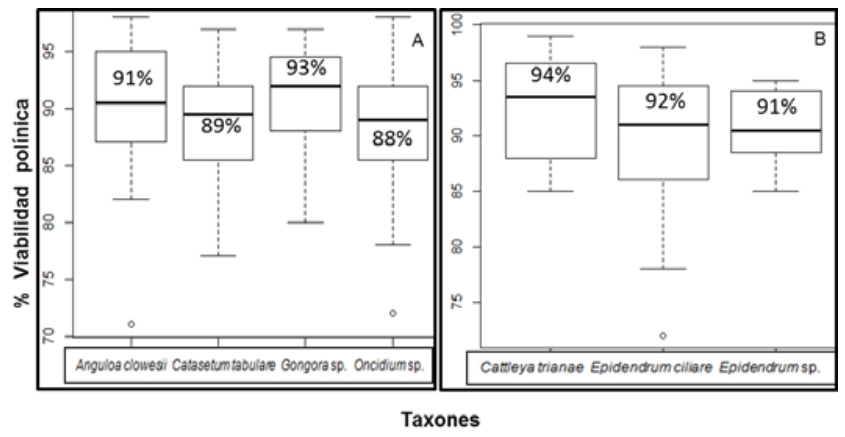

Figura 2. Comparación de medianas de la viabilidad polínica en los taxones de las tribus A. Cymbidieae y B. Epidendreae

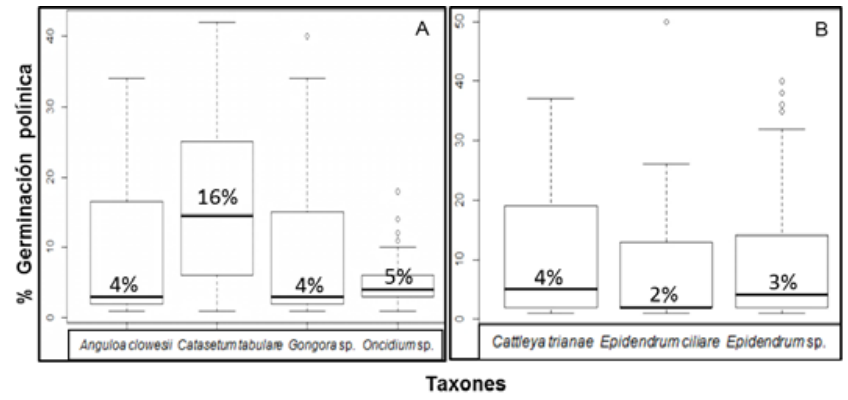

Figura 3. Comparación de la germinación del polen mediante las medianas obtenidas para cada taxón analizado en las tribus A. Cymbidieae y B. Epidendreae

a 6,356e-09 y 3 grados de libertad, hallándose diferencias significativas entre las medianas al comparar la germinación del polen en los cuatro taxones estudiados. 
En la tribu Epidendreae, con porcentajes de germinación bajos y un valor de $\mathrm{p}$ de 0,2023 y 2 grados de libertad, no hubo diferencias significativas entre las medianas, es decir, los taxones tenían la misma probabilidad de germinación, siendo Cattleya trianae la especie de mayor germinación, con $4 \%$, seguida de Epidendrum ciliare y Epidendrum sp., con 2 y $3 \%$, respectivamente (Figura 3B).

En la mayoría de taxones predominó un crecimiento reducido y lento de tubos polínicos que difícilmente logró superar dos veces la longitud del grano de polen, siendo esta la medida estándar de una elongación efectiva en polen germinado (Figura 4 A-B). En este estudio el polen de Oncidium sp., evidenció los mayores problemas a la hora de exhibir el tubo polínico a los tres días de evaluación (Figura 4C), a diferencia del comportamiento del polen de $A$. clowessi, en el cual se observó la presencia de una vacuola que actuó como una herramienta hidráulica impulsando la mayor parte del citoplasma hacia la punta del tubo polínico (Figura 4D). Por último, en la Figura 4E se observa un núcleo redondeado que al parecer corresponde a la célula vegetativa, el cual guio la punta del tubo polínico.

Respuesta germinativa en los tres medios de cultivo. Los ensayos de germinación evidenciaron los efectos positivos del medio M3 (basado en el uso de mucílago estigmático), con un porcentaje de germinación polínica que alcanzó el $18 \%$ a las 72 horas de evaluación. Usando los medios M1 y M2, cada uno suplementado con sacarosa al 1 y $10 \%$, respetivamente, hubo una menor respuesta en la emisión del tubo polínico, con un porcentaje de germinación de $3 \%$ en ambos medios (Figura 5A). Este resultado se ratificó con el análisis no paramétrico de comparación de medianas, el cual determinó diferencias altamente significativas entre las medianas del medio germinativo M3 y los medios M1 y M2 al evaluar los polinios de la tribu Cymbidieae, con un valor de $\mathrm{p}$ menor de 2,2e-16 y 2 grados de libertad.

Respecto a la tribu Epidendreae, la mayor respuesta germinativa se vio en el medio M3, con un $20 \%$ de polen susceptible de germinar, comparado con los medios M1 y M2, en los cuales se mantuvo el patrón de baja respuesta germinativa, con valores de 3 y $2 \%$, respectivamente (Figura 5B). En esta tribu los resultados obtenidos evidenciaron la existencia de diferencias altamente significativas (KruskalWallis: p menor a 2,2e-16 y 2 grados de libertad) entre las medianas correspondientes a la germinación de polen con los tres medios.

\section{Discusión}

Probablemente los altos porcentajes de viabilidad del polen exhibidos en este estudio se deben a que los polinios se usaron en estado fresco, inmediatamente después de ser extraídos de la antera, y se procesaron con acetocarmín glicerol al $2 \%$. En este sentido, Yuan, et al. (2018) también notaron que el almacenamiento del polinio a temperatura ambiente durante cuatro semanas preservaba las características del polen, pero a partir de ahí, este perdía funcionalidad. Asi mismo, al evaluar la viabilidad del híbrido Phalaeonopsis

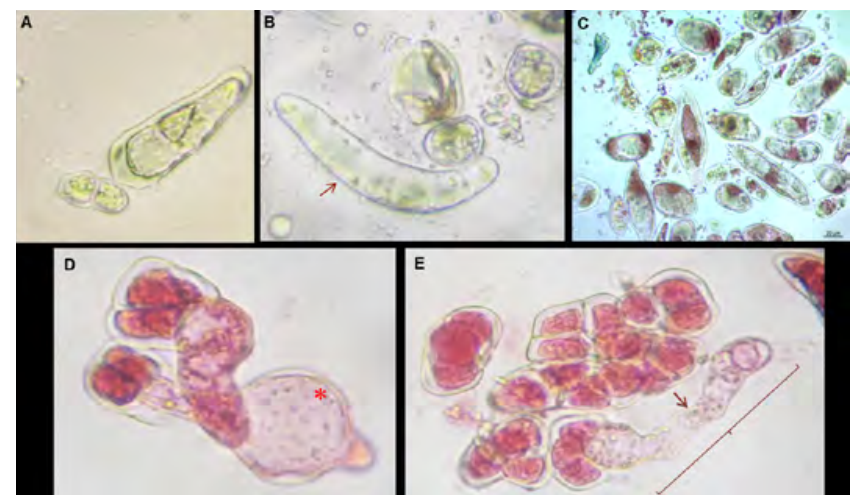

Figura 4. Germinación de las especies A y B. Gongora sp., C. Oncidium sp., y D y E. Anguloa clowessi, a las 12 horas de observación en medio M3.

*: fuerza impulsora de la vacuola; flecha: longitud del tubo polínico

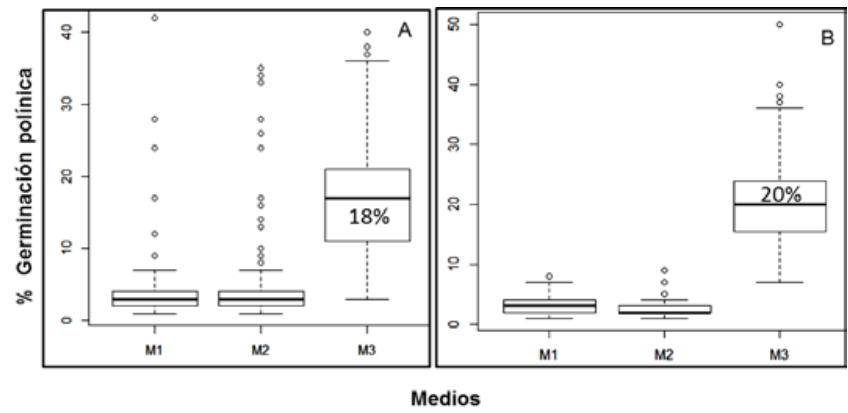

Figura 5. Comparación de medianas al evaluar la germinación del polen en tres medios de cultivo in vitro en A. polinios de la tribu Cymbidieae y B. polinios de la tribu Epidendreae

a diferentes temperaturas mediante tinción con TCC y germinación in vitro, estos autores reportaron que el polen había sido viable hasta por 40 semanas almacenándolo a $4{ }^{\circ} \mathrm{C}$, pero pasado este tiempo, era necesario almacenarlo a -20 y $-80^{\circ} \mathrm{C}$.

En términos generales, se cree que el polen de las orquídeas puede permanecer intacto y viable dentro del polinio durante periodos relativamente largos. Si se compara la viabilidad de los siete taxones estudiados con los de orquídeas con polinios menos compactados, la tendencia a conservar las características del polen durante largos periodos se mantiene. Es el caso de algunas orquídeas europeas de la subfamilia Orchidoideae con polinios séctiles, en las cuales también se han observado altos niveles de viabilidad reflejados en su coloración citoplasmática al emplear, no solo el colorante acetocarmín glicerol, sino, además, el azul de lactofenol (Neiland \& Wilcock, 1995). Al parecer, la alta viabilidad o la longevidad observada en orquídeas que exhiben y dispersan su polen a manera de agregados polínicos tiene un componente adaptativo, como lo señalan Johnson \& Edwards (2000), desarrollado como forma de contrarrestar el tiempo que puede transcurrir desde la captura del polinario por el polinizador hasta la entrega del polinio al estigma, lo cual puede tardar varios días. 
Por otra parte, al analizar la viabilidad del polen granular de Vanilla planifolia (subfamilia Vanilloideae) conservado durante dos meses, Borballa, et al. (2014) encontraron elevados porcentajes de polen viable con dos métodos de tinción, pero consideraron que se trataba de una sobreestimación de los resultados al emplear la tinción con carmín acético. En este sentido, se cree que la prueba de viabilidad con acetocarmín glicerol al $2 \%$ sería efectiva para determinar la calidad del contenido citoplasmático de orquídeas con polinios compactos, pero estos resultados deben sustentarse con el empleo de métodos más rigurosos de análisis, ya que, en ocasiones, con el carmín puede sobreestimarse la fertilidad del polen, apreciación que coincide con lo planteado por Towill \& Walster (2000) e Iglesias \& Tivo (2005).

En cuanto a la germinación in vitro, el comportamiento anormal del polen de Oncidium sp. observado en el proceso de su germinación, se debe a una extensa vacuolización y al aumento exagerado en el volumen de estos granos de polen. En un desarrollo normal, estos procesos ocurren después de la extensión del tubo polínico (Pacini, 1994); sin embargo, las vacuolas suelen desaparecer debido a la formación de un nuevo citoplasma o al almacenamiento de carbohidratos y lípidos, pero en el caso de Oncidium sp., no se observaron tubos correctamente definidos.

Ordóñez (2014) reconoce que el éxito de la germinación in vitro depende en gran medida del genotipo en evaluación. En Oncidium y otras orquídeas como Lycaste, Notylia, Coelogyne y Dendrobium, de floración masiva, existen antecedentes de autoincompatibilidad gametofítica, expresada en la abscisión de la flor mas no en la inhibición del crecimiento del tubo polínico (Johansen, 1990), lo cual contrasta con la escasa aparición de tubos polínicos o la falta de crecimiento en el presente estudio, factores considerados por Büyükkartal (2003) como una anomalía con respecto a la germinación in vitro, lo que podría explicar la falta de expresión y de dominancia de la punta del tubo polínico evidenciada al usar el medio germinativo M3.

Es posible que las variaciones en los porcentajes de germinación también estén relacionadas con la diferencia en la floración y la disponibilidad de polen en los distintos materiales (Garduño, et al., 2011). En este sentido, $C$. tabulare presentó un comportamiento peculiar, ya que se registraron mayores niveles de germinación con el polen recolectado en el mes de marzo que los porcentajes obtenidos con polen recolectado en la misma planta en el mes de septiembre, cuando la germinación decreció sorpresivamente, aun cuando se mantenían las condiciones controladas de incubación. Este hecho influyó en los resultados de expresión polínica en $C$. tabulare, aunque el polen de esta especie tuvo el mejor comportamiento (con un $16 \%$ de germinación). Por tal motivo, se considera conveniente hacer evaluaciones futuras en las que se determine si las condiciones ambientales están influyendo directamente en el adecuado desarrollo de la antera y el polen y en su función como progenitores. En este sentido, Kakani, et al. (2005) han mencionado que durante la floración el polen puede verse afectado por las altas temperaturas, ya que al ser liberado de las anteras pasa a ser una unidad funcional independiente, la cual está expuesta a los cambios medioambientales (Snow \& Spits, 1991).

El proceso de germinación de las orquídeas está mediado en gran parte por la formación de una gran vacuola en la célula vegetativa (Cocucci, 1973), lo que se evidenció en la germinación de $A$. clowessi, y, aunque la célula generativa no se observó claramente, en los polinios de las orquídeas se ha reportado su presencia en forma circular antes de emitirse el tubo polínico, y cómo una vez ingresa por este canal toma forma de huso (Pandolfi \& Pacini, 1995).

Excepto en $C$. tabulare, la germinación de los seis taxones restantes osciló en un rango de 3 a $5 \%$. Estos bajos porcentajes son acordes con el tiempo de evaluación empleado en este estudio, es decir, tres días de inmersión en los diferentes medios, el cual probablemente fue relativamente corto si se tiene en cuenta que bajo condiciones normales de germinación, el periodo entre la germinación del polen y la fecundación en las orquídeas es prolongado, por lo que el polen germinado y los tubos polínicos deben sobrevivir en los ovarios antes de entrar en los sacos embrionarios maduros durante un tiempo bastante prolongado. Tales períodos pueden ser de mínimo cuatro días, como en Gastrodia elata, o tardar 10 meses, como sucede en Vanda suavis (Arditti, 1992).

Aunque estos métodos de germinación son muy útiles y efectivos, sigue siendo difícil evidenciar el desarrollo total del tubo polínico in vitro, porque, al parecer, la mayoría de medios no logran satisfacer las necesidades fisiológicas de este tipo de polen con textura compacta. De allí que nuestra conclusión general coincida con la de la mayoría de trabajos, en los cuales se señala que, independientemente del medio y las condiciones empleadas en la germinación in vitro en orquídeas, esta es baja y lenta (Pritchard \& Prendergast, 1989).

Hay poca información sobre la germinación del polen agregado en polinios de la familia Orchidaceae. En el caso de la tribu Cymbidieae, se reportó la germinación en la especie Cymbidium elegans, en la cual Pritchard \& Prendergast (1989) encontraron poca significación estadística en la germinación comparada con especies que presentaban polinios organizados en másulas. Estos resultados coinciden con los bajos niveles de germinación hallados en este estudio en las especies $A$. clowessi, Oncidium sp. y Gongora sp. Con respecto a la tribu Epidendreae, no se han registrado estudios sobre la biología del polen, por lo cual los resultados de la presente investigación son un aporte importante para la comprensión de este proceso. Por otra parte, a nivel de familia, sí se conocen reportes acerca de la germinación de Orchidaceae frente a otras tribus, como es el caso de la especie Phalaenopsis aphrodite (tribu Vandeae), también con polinio compacto, en la cual se registró la germinación del polen a partir de los 60 días de exposición en medio líquido bajo condiciones controladas de incubación (Chen \& Fang, 2016). 
Por lo tanto, no es factible asegurar que la poca germinación de las tétradas polínicas en los taxones estudiados se relacione con fenómenos de esterilidad, como lo han reportado algunos autores en híbridos de orquídeas (Von Kichner, 1992), en tanto no se demuestre lo contrario con estudios in vivo basados en técnicas de polinización manual y evaluación directa de los niveles de germinación en estigmas receptivos. Según Rejón, et al. (2010), hay que tener en cuenta que los factores óptimos de la germinación en un medio in vitro nunca serán iguales a las germinaciones producidas en el pistilo y, por lo tanto, el porcentaje que se obtenga siempre será diferente al que en realidad se tiene en la flor in situ.

La viabilidad y el éxito germinativo son factores clave en la producción de frutos y en la dispersión efectiva de las semillas, lo cual influye en la permanencia y la propagación de las poblaciones, así como en la supervivencia a largo plazo de las especies (Cabrera, et al., 2010). Por ello, se deben continuar desarrollando investigaciones que mejoren las técnicas descritas hasta ahora. El presente análisis debe ser visto como una primera evaluación del potencial germinativo, sin descartar el potencial parental a nivel reproductivo de estas orquídeas, ya que es probable que el análisis in vitro no lo haya reflejado del todo debido a la alta especificidad y los complejos mecanismos de activación celular que controlan la comunicación e interacción de los gametos masculinos en los procesos de fertilización tan característicos de esta familia.

En cuanto al comportamiento de los tres medios de germinación, la mejor expresión del tubo polínico en las orquídeas de las tribus Cymbidieae y Epidendreae se logró con el medio M3, el cual puede hacer favorecido la germinación porque el estigma (de aproximadamente cuatro cavidades estigmáticas por individuo evaluado), ofreció suficiente concentración y suministro de minerales para los genotipos estudiados. Además del fluido estigmático, este medio de cultivo se caracterizó por la presencia de una base de agar al $1 \%$, la cual pudo haber contribuido a generar el soporte y la consistencia necesarios para la elongación del tubo polínico.

En este sentido, algunos autores afirman que en el estigma receptivo de las orquídeas existe una sustancia azucarada que recibe a los polinios y permite que los granos de polen germinen, pero aún se desconocen las propiedades y composición biológica de dicha sustancia. Lo que sí está claro es que las superficies estigmáticas han evolucionado de manera simultánea para adaptarse a las diferentes unidades de dispersión polínica (Nilsson, 1983). Inicialmente, el medio M3 se propuso como una opción frente a los medios convencionales de germinación evaluados a lo largo de esta investigación (alrededor de 15 medios; no se incluyen los datos), pero pasó a ser la propuesta óptima al dar resultados medianamente positivos considerando las dificultades de los medios M1 y M2 para estimular la emisión del tubo polínico.
Brewbacker \& Kwack (1963) determinaron el medio de cultivo adecuado en cerca de 86 especies de 39 familias diferentes, sin incluir polen de orquídeas, y comprobaron que la ausencia del ion calcio inhibía por completo el desarrollo del tubo polínico in vitro. Utilizando el medio BK (1963), Tsai \& Chang (2010) introdujeron una serie de modificaciones para mejorar las condiciones de germinación.

En este estudio, con el uso del medio modificado por Tsai \& Chang (2010), denominado medio M2, la germinación no superó el $3 \%$ en los taxones analizados, sin embargo, la especie C. tabulare evidenció una respuesta positiva en este medio, lo cual obedeció principalmente al estado de maduración del polen en el polinio y a factores ambientales asociados con el momento en que se tomó la muestra. Asimismo, la germinación del polen en el medio M1 a una temperatura de 25 a $27^{\circ} \mathrm{C}$ fue muy poca, tanto así que a los tres días de observación había cerca de un $3 \%$ de polen germinado en las dos tribus, lo que estaría relacionado con la baja concentración de ácido bórico en el medio $(0,1 \%)$ de Pritchard \& Prendergast (1989). Se sabe que el nivel de boro es muy importante para la germinación, ya que facilita la absorción de sacarosa y la producción de proteínas en el tubo del polen, por lo que no se descarta su uso en concentraciones mayores en estudios futuros (Kavand, et al., 2014).

Chen \& Fang (2016) introdujeron una variación en el medio modificado de Tsai \& Chang (2010) agregando extracto estigmático de la antera de Phalaenopsis aphrodite. Hasta el momento, la metodología de Chen \& Fang (2016) ha sido la única con la que se ha logrado evaluar la calidad del polen compacto en la subfamilia Epidendroideae. Su uso exitoso ratifica, igualmente, la importancia de incluir estigma en los medios artificiales de germinación cuando se emplean unidades compactas de orquídeas como modelo de estudio.

Uno de los medios universalmente empleados para evaluar el potencial germinativo de polen agregado en polinios incluye el uso de sacarosa en diferentes concentraciones. Rao \& Ong (1972) recomendaron concentraciones bajas (5\%) en polinios de orquídeas y concentraciones mucho más altas (20 a $30 \%$ ) en políadas de Mimosoideae y polinios de Asclepias. En este estudio también se hicieron ensayos preliminares de germinación con soluciones azucaradas en concentraciones de 5, 10, 20 y $30 \%$. Sin embargo, con las concentraciones bajas no se observó la presencia de tubos polínicos y con las mayores, la germinación fue relativamente baja, observándose tubos polínicos anormales y deformes, por la cual se descartó esta opción.

En la etapa final de los ensayos in vitro se decidió corroborar lo reportado por Chen \& Fang (2016) replicando su metodología con el uso de extracto estigmático de cada uno de los taxones en estudio. Pero pasados tres meses de inmersión en este medio líquido, no se observó una estimulación polínica significativa (no se incluyen los datos), lo cual podría deberse, principalmente, a la especie y al medio de germinación evaluados. Ello supone que las orquídeas no 
germinan fácilmente en condiciones in vitro, como sí ocurre con muchas especies con polen en mónade o tétrade, en las cuales esta técnica ha funcionado fácilmente y con rapidez, en parte porque los granos de polen de estas orquídeas no se asemejan a los de otras especies con polen en polinios, pues, además de presentar un tamaño bastante reducido y no tener aperturas, la forma de los polinios varía considerablemente en cada tribu dentro de la subfamilia Epidendroideae. Por último, los resultados ratifican la influencia positiva del estigma como un promotor de la germinación del polen de orquídeas in vitro.

\section{Conclusiones}

En los siete taxones estudiados la viabilidad superó el $85 \%$, a pesar del alto grado de compactación del polinio, lo que supondría una gran fertilidad en estos individuos, algo fundamental para garantizar el éxito en el proceso de fecundación.

La gran viabilidad polínica contrastó con la poca producción de tubos polínicos, lo que evidenció la presencia de complejos mecanismos de señalización celular en las vías que controlan la formación y la dominancia de la punta del tubo polínico, lo que hace de este grupo un modelo difícil de evaluar in vitro.

La efectividad significativa del medio M3 que, a diferencia de los otros medios, incluyó el mucílago del estigma propio de cada taxón evaluado, ratificó la dificultad en la germinación del polen de orquídeas en condiciones artificiales y la dependencia con respecto a las sustancias estimuladoras de la germinación del polen presentes en el estigma.

De los siete taxones evaluados que presentaron polen viable, la especie $C$. tabulare tuvo la mejor respuesta germinativa en condiciones in vitro, tanto en el medio M2 como en el M3. Pese a la complejidad de su proceso de floración, esta especie resultó ser un buen modelo biológico para la evaluación de la biología polínica (viabilidad y germinación in vitro) en los polinios de esta subfamilia.

Los resultados aquí reportados son importantes porque aportan información sobre los mecanismos de adaptación reproductiva de la subfamilia y sus implicaciones en la conservación de las especies. Dada la vulnerabilidad actual de gran parte de las orquídeas, es importante continuar incentivando el desarrollo de estudios sobre el tubo polínico que promuevan el conocimiento y la propagación de estas especies.

\section{Agradecimientos}

Los autores agradecen al Comité Central de Investigaciones de la Universidad del Tolima por la financiación de este proyecto, así como a los integrantes del Grupo GEBIUT.

\section{Contribución de los autores}

Las dos autoras participaron por igual en el desarrollo del estudio, así como en la redacción, revisión y aprobación final del documento. Adriana P. Bonilla-Sánchez estandarizó y desarrolló las metodologías en la fase de laboratorio, y analizó los resultados obtenidos en la investigación. Por su parte, Hilda R. Mosquera-Mosquera contribuyó con el análisis de los resultados y su discusión.

\section{Conflicto de intereses}

Los autores declaran no tener conflictos de intereses.

\section{Referencias}

Araméndiz T. H., Cardona A. C., Lugo T. E. (2013). Eficiencia de dos métodos para evaluar la viabilidad del polen de berenjena. Revista U.D.C.A Actualidad \& Divulgación Científica. 16: 351-358.

Arditti, J. (1992). Fundamentals of Orchid biology. New York: John Wiley \& Sons. p. 704.

Brewbaker, J.L. \& Kwack, B.H. (1963). The essential role of calcium ion in pollen germination and pollen tube growth. American Journal of Botany. 50: 859-865.

Büyükkartal, H.N. (2003). In vitro pollen germination and pollen tube characteristics in tetraploid red clover (Trifolium pratense L.). Turk J Bot. 27: 57-61

Cabrera, V.A., Dottori, N., Cosa, M.T. (2010). Germinación, éxito reproductivo y fenología reproductiva de Solanum chenopodioides (Solanaceae). Boletín de la Sociedad Argentina de Botánica, 45: 73-80.

Chen, J.C. \& Fang, S.C. (2016). The long pollen tube journey and in vitro pollen germination of Phalaenopsis orchids. Plant Reprod. 29: 179-88.

Díez, S.J.M. (2011). Guía de orquídeas silvestres de la montaña oriental leonesa. España: Editorial Grupo de acción local Montaña de Riaño. p. 248.

Damon, A. \& Nieto, G. (2012). A Guide to the Morphology of the Pollinia and Pollinaria of Orchids from the Biological Corridor Tacaná-Boquerón in Southeast México. Selbyana. 31: 5-39.

Dressler, R.L. (1981). The orchids: Natural history and classification. Cambridge, Massachusetts: Harvard University Press. p. 344.

Dressler, R.L. (1993). Phylogeny and classification of the orchid family. Portland, OR: Melbourne, Australia: Dioscorides Press. p. 314.

Freudenstein, J.V. \& Rasmussen, F.N. (1997). Sectile pollinia and relationships in the Orchidaceae. Plant Syst. Evol. 205: 125-146.

Garduño, N. A., Núñez, C.A., Pecina, V., Montero, V., Montes, N., González, M.M., Anaya, L.J.L. (2011). Desarrollo de un método eficiente para la germinación in vitro de polen de sorgo. Tropical and Subtropical Agroecosystems. 14: 901-906.

Hesse, M., Burns-Balogh, P., Wolff, M. (1989). Pollen morphology of the "primitive" epidendroid orchids. Grana. 28: 261-278.

Johansen, B. (1990). Incompatibility in Dendrobium (Orchidaceae). Bot. J. Linn. Soc. 103: 165-196.

Johnson, S.D. \& Edwards, T.J. (2000). The structure and function of orchid pollinaria. Plant Syst. Evol. 222: 243-269.

Kakani, V.G., Reddy, K.R., Koti, S., Wallace, T.P., Prasad, V.V.P., Reddy, V.R., Zhao, D. (2005). Differences in in vitro pollen germination and pollen tube growth of cotton cultivars in response to high temperature. Annals of Botany. 96: 59-67. 
Kavand, A., Ebadi, A., Shuraki, D.Y., Abdosi, V. (2014). Effect of calcium nitrate and boric acid on pollen germination of some date palm male cultivars. Euro. J. Exp. Bio. 4: 10-14.

Mosquera, H.R., Valencia, R.M., Acedo, C. (2019). Variation and evolutionary transformation of some characters of the pollinarium and pistil in Epidendroideae (Orchidaceae). Pl. Syst. Evol. 305: 353-374.

Ordóñez, B. (2014). Determinación de la viabilidad y fertilidad del polen. Lima, Perú: Centro Internacional de la Papa (CIP). p. 8.

Pacini, E. (1994). Cell biology of anther and pollen development. In: E.G. Williams, A.E Clarke y R.B Knox, editors. Genetic control of self-incompatibility and reproductive development in flowering plants (289-308). Dordrecht: Kluwer Academic Publishers.

Pandolfi, T. \& Pacini, E. (1995). The pollinium of Loroglossum hircinum (Orchidaceae) between pollination and pollen tube emission. Plant Syst. Evol. 196: 141-151.

Pritchard, H. \& Prendergast, F. (1989). Factors influencing the germination and storage characteristics of orchid pollen. In H. Pritchard (Ed.), Modern Methods in Orchid Conservation. Cambridge: Cambridge University Press.
Rao, A.N. \& Ong, E.T. (1972). Germination of Compound Pollen Grains. Grana. 12: 113-120.

Rothacker, E.P. (2007). The primitive Epidendroideae (Orchidaceae): Phylogeny, character evolution and the systematic of Psilochilus (Triphoreae). The Ohio State University. p. 448.

Rejón, J.D., Suárez, C.G., Alché, J.D., Casastro, A.J., Rodríguez, M.I. (2010). Evaluación de diferentes métodos para estimar la calidad del polen en distintos cultivares de olivo (Olea europea L.). Polen. 20: 61-72.

Singer, R., Gravendeel, B., Cross, H., Ramírez, S. (2008). The use of orchid pollinia or pollinaria for taxonomic identification. Selbyana. 29: 6-19.

Snow, A. \& Spits, T. (1991). Pollen vigor and the potential for sexual selection in plants. Nature. 352: 796-797.

Tsai, Y.C. \& Chang, C. (2010). The investigation of floriculture characteristics, chromosome numbers and in vitro pollen germination of Phaius tankervilleae and its allied genera. Hortic NCHU. 35: 99-112.

von Kirchner, O. (1922). Uber Selbstebestaubung bei den Orchideen. Flora. 115: 1003-129. 